\title{
Challenges for Wireless Mesh Networks to provide reliable carrier-grade services
}

\author{
D. von Hugo and N. Bayer \\ Deutsche Telekom AG Laboratories, 64295 Darmstadt, Germany
}

\begin{abstract}
Provision of mobile and wireless services today within a competitive environment and driven by a huge amount of steadily emerging new services and applications is both challenge and chance for radio network operators. Deployment and operation of an infrastructure for mobile and wireless broadband connectivity generally requires planning effort and large investments. A promising approach to reduce expenses for radio access networking is offered by Wireless Mesh Networks (WMNs). Here traditional dedicated backhaul connections to each access point are replaced by wireless multi-hop links between neighbouring access nodes and few gateways to the backbone employing standard radio technology. Such a solution provides at the same time high flexibility in both deployment and the amount of offered capacity and shall reduce overall expenses.

On the other hand currently available mesh solutions do not provide carrier grade service quality and reliability and often fail to cope with high traffic load. EU project CARMEN (CARrier grade MEsh Networks) was initiated to incorporate different heterogeneous technologies and new protocols to allow for reliable transmission over "best effort" radio channels, to support a reliable mobility and network management, self-configuration and dynamic resource usage, and thus to offer a permanent or temporary broadband access at high cost efficiency.

The contribution provides an overview on preliminary project results with focus on main technical challenges from a research and implementation point of view. Especially impact of mesh topology on the overall system performance in terms of throughput and connection reliability and aspects of a dedicated hybrid mobility management solution will be discussed.
\end{abstract}

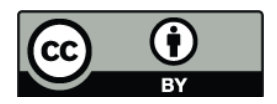

Correspondence to: D. von Hugo (dirk.von-hugo@ telekom.de)

\section{Introduction}

According to Wikipedia (2009) "a Wireless Mesh Network (WMN) is a communications network made up of radio nodes organized in a mesh topology. Wireless mesh networks often consist of mesh clients, mesh routers and gateways." Within the last few years WMNs try to fulfil emerging market demands for lowering capital and operational expenses of access networks which generally increase with degree of coverage, capacity, and provided quality. Flexibility and scalability of such WMNs is beneficial especially for broadband wireless city scenarios or deployments for temporal events or in case of emergency situation which overload and stress traditional networks. The technology of radio access on the other hand is steadily evolving towards higher data rates and frequency efficiency such that the (wired) backhaul connectivity for access networks is a major cost factor for operators. Here mesh networking technology represents a cost effective and efficient alternative as several gateways to the fixed backbone can be replaced by the mesh. A large still remaining challenge is the lack of support for carrier grade service quality and reliability within current mesh solutions.

To achieve a substantial performance improvement for Infrastructure WMNs (Akyildiz, 2005) EU project CARMEN (2009) has specified an architecture providing operators with a complementary infrastructure which supports delivery of carrier grade services with low operational maintenance effort and at affordable equipment cost, allowing at the same time for a high flexibility in deployment. Employment of heterogeneous technologies such as IEEE802.11 (WiFi), 802.16 (WiMAX), DVB (Digital Video Broadcasting), or future radio access technologies (RATs) allows to adapt the system to different environments and user demands ranging from provision of radio coverage for emergency communication to deployment of a broadband wireless city network which reflects basically a capacity limited scenario.

Developing a standardized abstraction mechanism enables the execution of technology-independent higher layer functionalities for a variety of wireless standards. These 


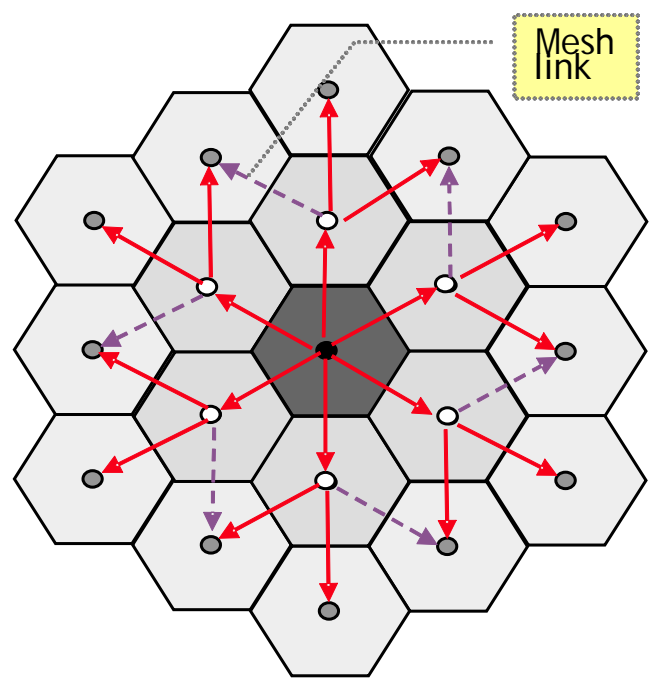

Fig. 1. Exemplary reference mesh scenario with 19 meshed Access Points and a central Gateway. Mesh links additional to links within a tree structure are indicated by dashed arrows.

functional modules cover both control tasks such as selfconfiguration and monitoring as well as routing related ones, e.g. capacity handling and mobility support. The concept shall enable mobility, uni- and bi-directional links, uni- and multicast services, resource awareness, ease of use, and selfoptimization (Azcorra et al., 2009).

An illustrative example of a reference deployment with 19 Access Points (APs) per GateWay (GW) is shown in Fig. 1. Here the main challenges of mesh networking can be seen: Requiring only one connection to the backhaul instead of 19 within a traditional approach the mesh concept can lower costs but the so-called " $1 / \mathrm{n}$ problem" will degrade the system throughput with increasing amount of nodes and hops. E.g. each intermediate mesh point (denoted by a white dot) has to forward aggregated traffic of up to three APs additional to data emerging from users within the own cell area. The gateway here receives six times such an accumulated traffic which may result in major congestion.

On the other hand each access point is concurrently connected to at least two other nodes allowing for a traffic rerouting in case a path becomes unusable. This may occur due to link outage or reduced available capacity because of adverse propagation conditions including interference. Also traffic shaping or load balancing methods can be applied more easily to a mesh structure in order to control a flexible transmission resource usage efficiently. A capacity handling mechanism considering QoS constraints of traffic as well as a mobility module will support reliable provision of carriergrade triple-play services (data, audio, and video). Mobility in terms of changing APs as traffic source or destination may also occur without actual movement of the end User Terminal (UT). Proper planning and monitoring with central coordination will ensure a reliable system performance.
Furthermore the proposed concept shall guarantee scalability (increasing or reducing number of nodes and traffic volume), flexible deployment and operation (within different environments or for various usage scenarios), cost efficiency, adaptive re-configurability (to limit operational effort to a minimum), and inherent security (considering risks due to such a dynamic arrangement with many-to-many relations especially).

The rest of the paper is structured as follows: the architecture of CARMEN project based on corresponding design objectives is explained by an exemplary node and network specification in Sect. 2. Efficient and flexible media resource usage in terms of MAC scheduling providing means for reliable communication is handled in Sect. 3. How a granted QoS and capacity for E2E connectivity is provided via coordinated Routing, Capacity Handling, and Mobility Management improving the mesh is described in Sect. 4. Section 5 finally summarises this contribution by providing a discussion and conclusion.

\section{Architecture}

To achieve the above mentioned goals of the project an improved mesh architecture has been specified which shall address a flexible and cost effective deployment of a radio access network supporting fixed and mobile users - also for temporary operation (city/emergency scenarios). To enable incorporation of different radio technologies a link layer (Layer 2, L2) interface abstraction is specified and improvements for medium access like a new MAC scheduling are considered (see Sect. 3). Modular network functions for routing, capacity-handling, mobility, and self-configuration are designed which make use of the abstracted L2 information to interoperate efficiently and result in optimal performance for any situation. In Fig. 2 the network components are shown within an exemplary network configuration. They are classified as three different CARMEN entities, namely AP (Access Point), MP (Mesh Point), GW (GateWay), which serve the (traditional) User Terminals (UTs). All network entities have an abstract InterFace (IF) layer which provides support for RATs as e.g. WLAN, WiMAX, DVB, and any potential future new access technology. The functionalities on routing layer which include capacity handling and mobility support communicate with and via the abstract IF, control functionalities for self-configuration and monitoring make use of both abstract IF and routing information.

A draft overview on different functional blocks and their interworking is given in Fig. 3 where the specific node architecture is depicted. For each procedure during the operation of the mesh network a set of message sequence charts has been specified describing tasks and functions of each functional module as well as the messages exchanged between them. 


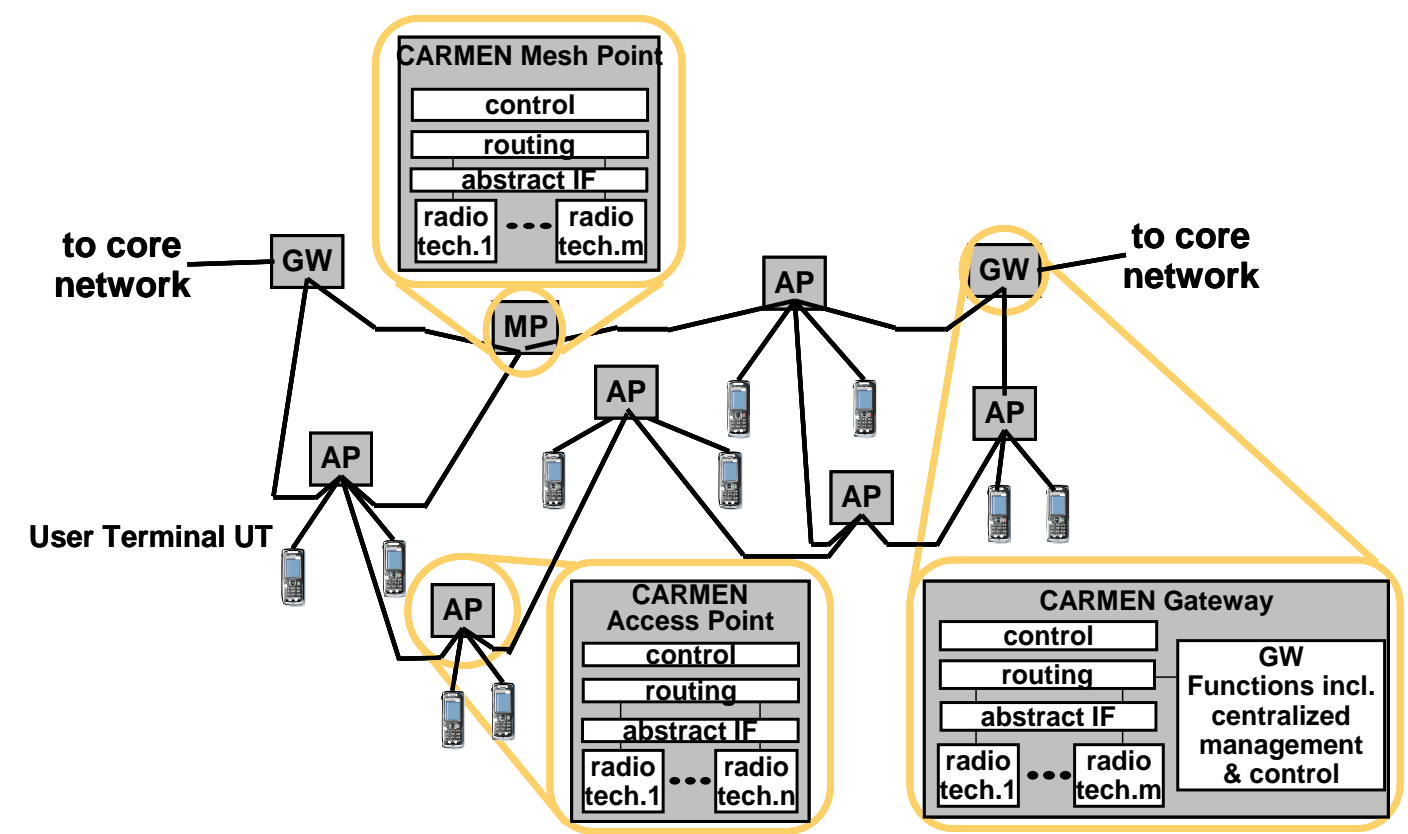

Fig. 2. Exemplary configuration of a CARMEN network with its mesh network entities.

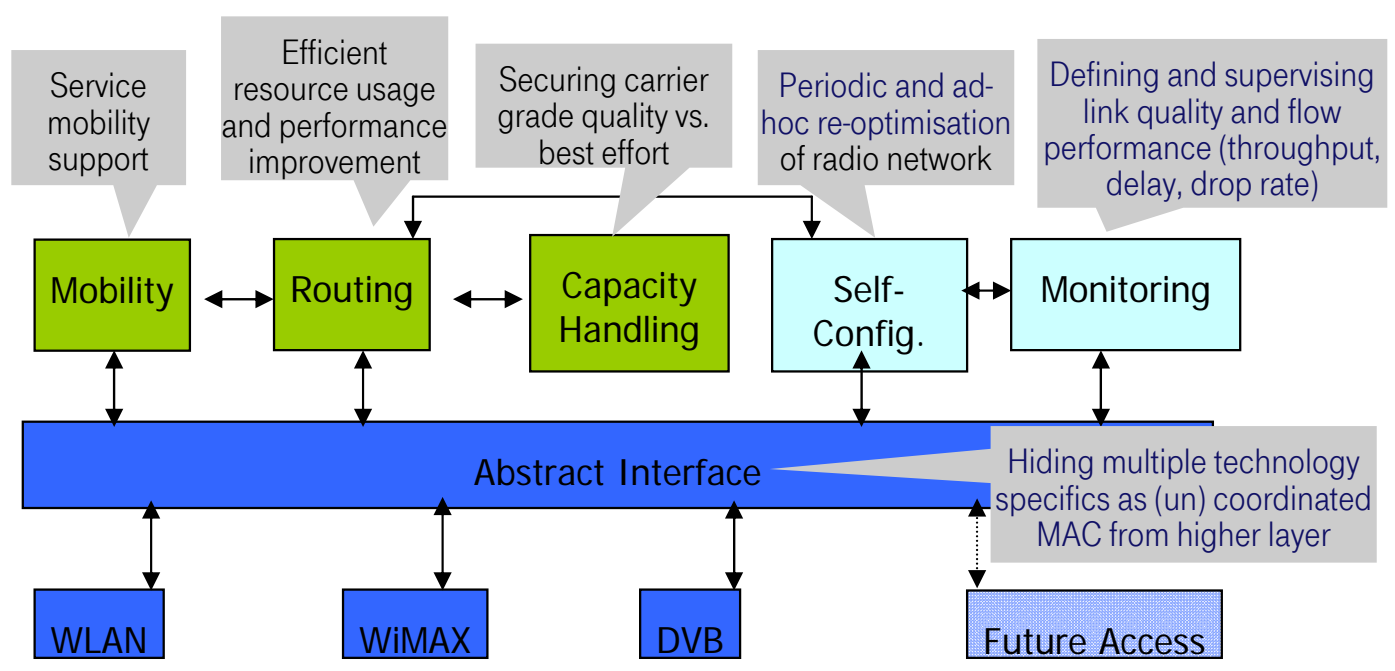

Fig. 3. CARMEN abstraction within each node to enable key functionalities.

Towards providing mobility support the approach includes a seamless, capacity-aware per-flow mobility management for end user terminals with or without a standard IEEE 802.21 extension enabling the network to make up a new connection before breaking the existing one. Details will be described in Sect. 4.

\section{Efficient and flexible media resource usage}

Aim of the effort to adapt mechanisms for access of different entities and interfaces to the common shared radio medium is to enable an efficient and flexible media resource usage within mesh networks. Mainly two effects are responsible for degradation in transmission quality: interference and packet collisions.

Transmission quality of uncoordinated access in multi-hop mesh networks degrades with growing amount of nodes due to high in- and out-of band interference. On the one hand the frequency selectivity of the antennas of simple mesh nodes is limited thus increasing signal interference by neighboring frequency channels, on the other hand a spatially highly directional antenna pattern complicates connectivity between mesh nodes, but a broad beam width allows distant mesh 


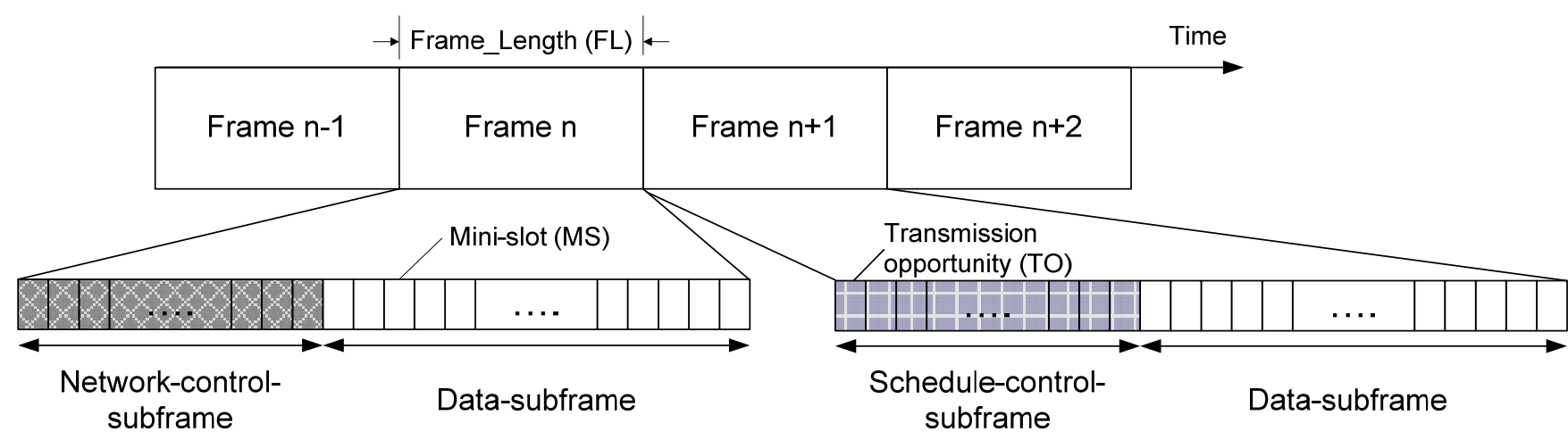

Fig. 4. General mesh TDMA frame structure.

nodes using the same channel to interfere. A correspondingly degraded Signal-to-Interference (S/I) ratio may exceed the threshold required for proper operation of a higher order modulation. To overcome these problems CARMEN uses a centralized network monitoring and management architecture which keeps track of the interference in the network and re-configures the channels in case the $\mathrm{S} / \mathrm{I}$ has crossed a predefined threshold.

Packet collisions and subsequent loss of data and signaling are caused by random access protocol (Carrier Sensing Multiple Access - Collision Avoidance: CSMA/CA) as is in use for WiFi (e.g. IEEE802.11). A proper scheduling which includes methods for resource request and coordination, policies and rules for assignment, and the consideration of resource management across multiple hops can ensure transmission timing of signalling messages and data packets in a collision free manner and thus significantly improve the provided service quality. Hence, CARMEN proposes an IEEE 802.11-based coordinated MAC technology which will be presented in the following.

The coordinated MAC technology adopts a distributed coordinated media access mechanism on top of the IEEE 802.11 standard. The motivation of this approach is to overcome the problems with the random access protocol of the IEEE 802.11 standard in multi-hop networks. Compared to IEEE 802.11 standard, it is expected that a TDMA-based MAC protocol is better suited for providing QoS to real-time applications. Among several reasons, most important is that the access to the medium is controlled and resources are explicitly reserved. Additionally, in TDMA systems, the channel for the control message exchange is separated from that for data transmission, which lets the data transmissions continue without being affected by the contention in the control channel. Another advantage of a TDMA system is that a single request message can be used to allocate multiple slots continuously, which is not possible in IEEE 802.11. Based on these reasons, a coordinated MAC protocol is proposed, which uses a TDMA frame structure to manage channel access and a distributed scheduler to coordinate the collisionfree access to the transmission slots of the frame.
The general TDMA frame structure has been adopted from the IEEE 802.16 mesh standard and is depicted in Fig. 4. The length of the TDMA frame is defined by the Frame_Length (FL) parameter (e.g. $4 \mathrm{~ms}, 10 \mathrm{~ms}$ ) and the frame is divided into the control-subframe and the data-subframe.

Slots in the control-subframe are called Transmission Opportunities (TOs) and they are used only for the transmission of signalling messages. The control-subframe serves several functions:

- The network-entry in which specific slots can be used by new nodes to join the mesh network

- Periodic broadcasting of network configuration messages to distribute information to the entire mesh network

- The transmission of signalling messages to schedule resources in the data-subframe

Slots in the data-subframe are called mini-slots (MSs) and they are mainly used for the transmission of data packets. The scheduling mechanism is responsible for allocation and reservation of MSs in the data-subframe. A schedule allocation in the data-subframe consists of one or more MSs. A series of mini-slots allocated for a mesh station is called a burst. Within a burst, a station can send one ore more MAC packet data units (PDUs).

Using the coordinated distributed scheduler, MPs compete for bandwidth based on the two-hop extended neighbourhood of the two communicating MPs, where a neighbourhood includes all the one-hop neighbours of a given MP as shown in Fig. 5. Coordinated distributed scheduling allows mesh nodes to transmit distributed scheduling signalling messages requesting bandwidth in the controlsubframe in a collision-free manner.

In the distributed scheduler, a three-way handshake mechanism is used to reserve bandwidth for data transmission between two MPs, regardless of direct communication or communication with the GW. Messages of this handshake are transmitted in the schedule-control-subframe. In order to 


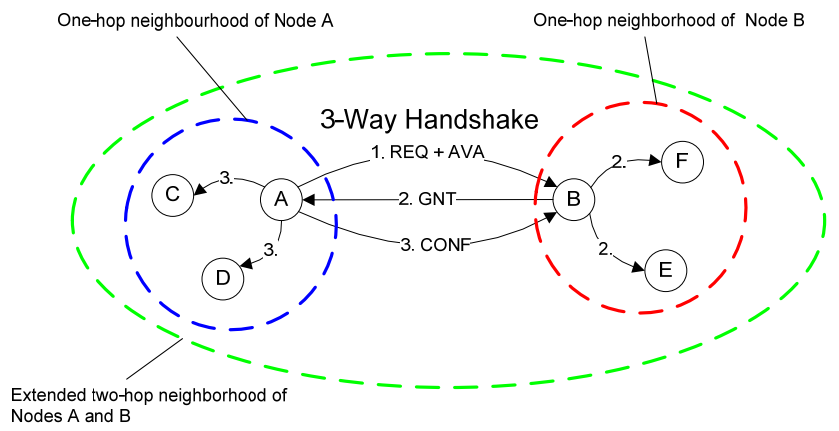

Fig. 5. Three-way handshake of the distributed scheduler.

explain the three-way handshake method, the example network shown in Fig. 5 will be used. If node A has data to send to node $\mathrm{B}$, node $\mathrm{A}$ initiates the three-way handshake by sending a request (REQ) including its availability (AVA) to node $\mathrm{B}$, which mainly lists the required bandwidth and available MSs of node A. All one-hop neighbours of node A, namely nodes $\mathrm{C}$ and $\mathrm{D}$, also receive this message and mark the listed MSs as unavailable to avoid assigning MSs multiple times. In response, Node B chooses enough MSs from that list to fulfil the bandwidth requirement and will reply with a grant (GNT) listing the MSs to be used for communication between nodes A and B. All the nodes in the one-hop neighbourhood of node $\mathrm{B}$, namely nodes $\mathrm{E}$ and $\mathrm{F}$, also receive the GNT and mark these MSs as unavailable. In turn, node A sends a confirmation (CONF) (which is simply a copy of the GNT) to acknowledge the receipt of the original GNT. Upon hearing the CONF message, all the nodes in the one-hop neighbourhood of node $\mathrm{A}$, nodes $\mathrm{C}$ and $\mathrm{D}$, mark these MSs as unavailable. After transmitting the CONF message, node A can begin sending data using the allocated MSs. Through the three-way handshake method, the extended twohop neighbourhood is defined as the area in which MSs may not be reused. However, stations are free to reuse resources outside of this area.

\section{Cooperation of message transfer functionalities}

To grant the actual QoS and provide enough capacity for E2E session connectivity between users within and/or outside the mesh network the higher layer message transfer modules are specified. These functionalities for Routing, Capacity handling, and Mobility management have to cooperate and synchronize their effort to achieve optimum performance in terms of scalability, overhead, responsiveness, resilience, etc. Routing for a dynamic mesh topology shall optimize traffic path lengths to reduce delay and resource consumption. Here a strong interoperation with Capacity Handling reserving service specific paths throughout the mesh is required.

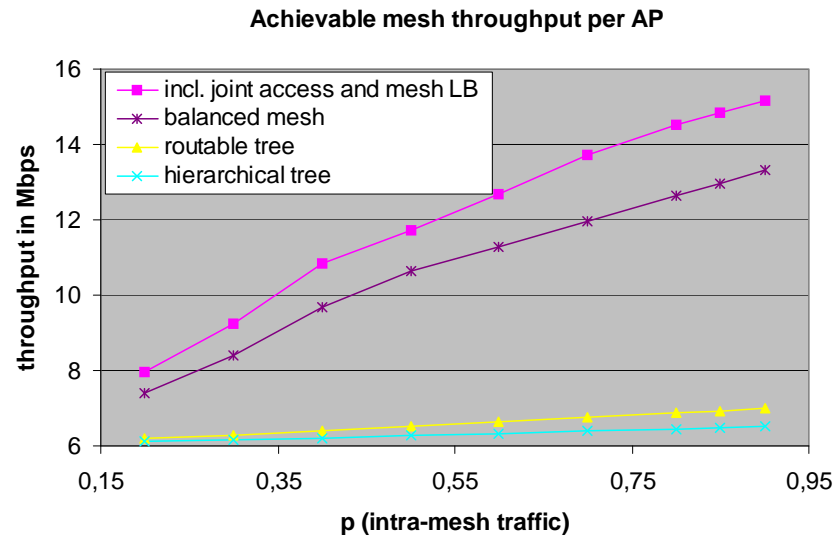

Fig. 6. Comparison of results for optimally balanced mesh routing (hop amount agnostic) vs. non-mesh structure (with/without routing ability) and joint mesh and access load balancing (mesh link of $18 \mathrm{Mbps})$

A network-based (local) Mobility Management shall support terminals movement but also decide on handover execution for network reasons e.g. load balancing between adjacent cells.

To achieve real seamless connectivity with "Make Before Break" handover preparation (anticipated movement) an approach based on IETF L3-protocol Proxy Mobile IP (Gundavelli et al., 2008) is proposed using UT's standard IEEE 802.21(L2) information. Here it is assumed that the correspondent node of the ongoing session of an active UT can be reached via the gateway acting as a local home agent or LMA (Local Mobility Anchor) to control the UT's actual location.

On the other hand a delay tolerant "Break Before Make" in case of accidental loss of connectivity (i.e. without a priori notification) can be supported by a direct inter-AP signaling and data forwarding to prevent extensive message load. This procedure is especially also advantageous in case of intramesh communication. Instead of exchanging location information via the LMA/gateway the next hop neighbours are "flooded" and intermediate packets are forwarded between old and new nodes or points of attachment. For a high node density and in vicinity of the gateway, however, this may result in unduly high signalling load. An analysis has been carried out to identify trade-off between both approaches resulting in an integrated CARMEN solution with correspondingly tuned parameters allowing for routing optimization.

A capacity-aware handover decision has to consider both currently available access and mesh link capacity to fully exploit mesh topology by coordinated load balancing. An overall throughput enhancement in the range between $33 \%$ and $110 \%$ was calculated exemplarily for the 19 node configuration shown in Fig. 1. The improvement depends on the traffic characteristics in terms of intra- and extra-mesh portion, i.e. the probability of internal and external location of the session 
endpoint. The details of these considerations are described in the following section.

\section{Exemplary mesh capacity improvement thanks to load balancing}

Based on the observation that the average mesh network throughput is mainly limited by the capacity of inter-node mesh links and depends on the percentage $p$ of intra-mesh traffic in relation to the overall traffic amount a calculation of the achievable mesh throughput was calculated. Assuming the reference scenario with a GW/AP ratio of 1/19 (Fig. 1), a symmetrical traffic characteristic and bidirectional links the access throughput per cell amounts to $44 \%$ and $76 \%$ of mesh link capacity for $p=20 \%$ and $80 \%$, respectively, as can be seen from Fig. 6 depicting the throughput in Mbps vs. the probability $p$ (of intra-mesh traffic) for $18 \mathrm{Mbps}$ mesh capacity. Here a mesh-internal route balancing was assumed to balance link loads for non-real time (minimum hop) constraint traffic (intelligent load-dependent routing). Additionally a common Load Balancing (LB) of access and mesh link capacity (e.g. by increasing access coverage of APs with low forwarding load and vice versa) and a homogeneous traffic distribution throughout the mesh area was considered. The improvement compared to a tree (non-mesh) structure amounts to $27 \%$ and $99 \%$ (again for $p=20 \%$ and $80 \%$, respectively).

An analytical outcome for the reference scenario (Fig. 1) is that an optimal load for $p>0.48$ is possible given as the average capacity $C$ of each AP as a function of the capacity $C$ of a mesh link and $p$ by:

$C_{\mathrm{AP}}=1 / 3 \frac{C_{\text {meshlink }}}{1-[\sqrt{(190 / p+2)}-3] p / 19}$

Further capacity increase by introducing additional mesh links (e.g. to establish an alternative link to the APs at the corner of the overall hexagonal structure with long range and thus lower capacity - links) is achievable for traffic characteristics exhibiting a large proportion of intra-mesh communications. The outcome of corresponding ongoing investigations will be dealt with in a subsequent paper.

\section{Discussion and conclusion}

In this paper the approach to improvement of a Wireless Mesh Network (WNM) architecture by functional modules was described which allow for control and subsequent reduction of congestion probability and vulnerability. Together with the implementation of new MAC scheduling concepts enabling reliable transmission over "best effort" radio channels under adverse propagation conditions as is the case for e.g. unlicensed WLAN frequencies and the introduction and proper design of functionalities which support mobility and network management, facilitate self-configuration, and dynamically grant efficient resource assignment independent of actually employed radio technologies, thus the provision of carrier-grade services will greatly be enhanced.

A practical demonstration and verification of CARMEN capabilities via prototypical implementation in a testbed via measurements and real applications is currently under preparation.

Acknowledgements. The authors thank their colleagues cooperating within the EU project CARMEN. The research leading to these results has received funding from the European Community's Seventh Framework Programme (FP7/2007-2013) under grant agreement no 214994. The views and conclusions contained here are those of the authors and should not be interpreted as necessarily representing the official policies or endorsements, either expressed or implied, of the CARMEN project or the European Commission.

\section{References}

Akyildiz, I., Wang X., and Wang, W.: Wireless mesh networks: a survey, Computer Networks, 47(4), 445-487, 2005.

Azcorra, A., Banniza, T., Chieng D., Fitzpatrick, J., von Hugo, D., Natkaniec, M., Robitzsch, S., and Zdarsky, F.: Supporting carrier grade services over wireless mesh networks - the approach of the European FP-7 STREP CARMEN, IEEE Communications Magazine, 2009 April 2009.

CARMEN: CARrier grade MEsh Networks, http://www. ict-carmen.eu/, retrieved October 2009.

Gundavelli, S., Leung, K., Devarapalli, V., Chowdhury, K., and Patil, B.: Proxy Mobile IPv6, RFC 5213, IETF, August 2008.

Wireless mesh network: http://en.wikipedia.org/wiki/Wireless mesh_network, retrieved October 2009. 